\title{
Mean field strategies induce unrealistic non-linearities in calcium puffs
}

\author{
Guillermo Solovey ${ }^{1}$, Daniel Fraiman ${ }^{2}$ and Silvina Ponce Dawson ${ }^{3}$ \\ 1 Laboratory of Mathematical Physics, The Rockefeller University, New York, NY, USA \\ 2 Departamento de Matemática y Ciencias, Universidad de San Andrés, Buenos Aires, Argentina \\ ${ }^{3}$ Departamento de Física, Facultad de Ciencias Exactas y Naturales - Universidad de Buenos Aires, Ciudad Universitaria, Buenos Aires, Argentina
}

\section{Edited by:}

Guillermo A. Cecchi, IBM Watson

Research Center, USA

\section{Reviewed by:}

Markus A. Dahlem, Technische Universitaet Berlin, Germany

Irina Baran, Carol Davila "University of

Medicine and Pharmacy," Romania

\section{*Correspondence}

Guillermo Solovey, Laboratory of Mathematical Physics, The Rockefeller

University, 1230 York Avenue,

New York, NY 10065, USA.

e-mail:gsolovey@rockefeller.edu
Mean field models are often useful approximations to biological systems, but sometimes, they can yield misleading results. In this work, we compare mean field approaches with stochastic models of intracellular calcium release. In particular, we concentrate on calcium signals generated by the concerted opening of several clustered channels (calcium puffs). To this end we simulate calcium puffs numerically and then try to reproduce features of the resulting calcium distribution using mean field models were all the channels open and close simultaneously. We show that an unrealistic non-linear relationship between the current and the number of open channels is needed to reproduce the simulated puffs. Furthermore, a single channel current which is five times smaller than the one of the stochastic simulations is also needed. Our study sheds light on the importance of the stochastic kinetics of the calcium release channel activity to estimate the release fluxes.

PACS: 82.33.-z, 02.30.Jr, 87.17.-d, 87.16.Vy, 87.16.A-

Keywords: calcium signals, puffs, stochastic model, mean field model

\section{INTRODUCTION}

An important problem in different areas of science is to relate phenomena that occur at different scales. In biology, an observed behavior at a certain level of organization is often the result of interactions at a lower level, with very different physical and temporal scales. Calcium $\left(\mathrm{Ca}^{2+}\right)$ signals are an extraordinary example of a multiple scale biological system suitable for being studied with different experimental techniques, each of which focus on a particular temporal and spatial scale. Therefore, it is important to understand the interactions of processes occurring at different scales and how to infer properties at unobserved scales from experimental observations. In this respect, mathematics is invaluable (Cohen, 2004).

$\mathrm{Ca}^{2+}$ ions are important signaling elements that regulate a large variety of cellular functions such as fertilization, proliferation, development, learning and memory, contraction and secretion (Berridge et al., 2000). The versatility of $\mathrm{Ca}^{2+}$ as a intracellular cell messenger is possible because cells have mechanisms to control efficiently and precisely the distribution of $\mathrm{Ca}^{2+}$ in space and time. Within cells, $\mathrm{Ca}^{2+}$ is kept at high concentrations in stores such as the endoplasmic reticulum (ER). Release of $\mathrm{Ca}^{2+}$ from the ER occurs through specialized channels among which inositol triphosphate $\left(\mathrm{IP}_{3}\right)$ receptors play a most relevant role (Foskett et al., 2007). $\mathrm{IP}_{3}$ Rs are spatially organized in clusters located on the membrane of the ER and separated by a few microns (Yao et al., 1995). The open probability of $\mathrm{IP}_{3}$ Rs depends on both the $\mathrm{IP}_{3}$ and cytosolic $\mathrm{Ca}^{2+}$ concentration ([C $\left.\mathrm{Ca}^{2+}\right]$; Taylor, 1998; Patel et al., 1999). A key feature is the well-established biphasic action of $\mathrm{Ca}^{2+}$ in both facilitating and inhibiting the opening of $\mathrm{IP}_{3} \mathrm{Rs}$. For relatively low $\left[\mathrm{Ca}^{2+}\right]$, the $\mathrm{Ca}^{2+}$ released by one channel increases the open probability of neighboring channels, whereas at high $\left[\mathrm{Ca}^{2+}\right]$ it favors a closed state of the channel (Iino, 1990; Bezprozvanny et al., 1991; Finch et al., 1991; Mak et al., 1998; Ramos-Franco et al., 1998). This dependence of the open probability of $\mathrm{IP}_{3} \mathrm{Rs}$ on cytosolic $\mathrm{Ca}^{2+}$ creates communication between channels. As a result of the spatial organization of channels and the process of $\mathrm{Ca}^{2+}$-induced $\mathrm{Ca}^{2+}$-release (CICR), cytosolic $\mathrm{Ca}^{2+}$ signals display a hierarchical spatiotemporal organization which in some cells (e.g., oocytes) have length scales that span over six orders of magnitude (Callamaras and Parker, 1994; Mak and Foskett, 1997; Shuai and Jung, 2003a). The hierarchy of $\mathrm{Ca}^{2+}$ signals go from local ones such as "blips" $\left(\mathrm{Ca}^{2+}\right.$ release through a single $\left.\mathrm{IP}_{3} \mathrm{R}\right)$ and "puffs" $\left(\mathrm{Ca}^{2+}\right.$ release through several IP $\mathrm{Rs}$ in a cluster; Sun et al., 1998; Bootman et al., 1997; Yao and Parker, 1991; Parker et al., 1996) to waves that propagate globally across the cell by successive cycles of CICR and $\mathrm{Ca}^{2+}$ diffusion between clusters (Yao and Parker, 1991; Parker et al., 1996; Callamaras et al., 1998; Sun et al., 1998). These signals have been observed using fluorescence microscopy and $\mathrm{Ca}^{2+}$ sensitive dyes (Bootman et al., 1997; Callamaras et al., 1998; Sun et al., 1998). However, fluorescence microscopy experiments cannot follow the kinetics of individual IP ${ }_{3} \mathrm{Rs}$ or the detailed $\mathrm{Ca}^{2+}$ dynamics within a cluster of channels in most cell types. The spatial resolution of conventional light microscopy is limited by diffraction. This determines that features below the $250-\mathrm{nm}$ length scales are unobservable in typical confocal images. This limit can be beaten using super-resolution techniques. Techniques of this type have been applied to $\mathrm{Ca}^{2+}$ signals using total internal reflection fluorescence (TIRF) and a fast CCD (Smith and Parker, 2009; Parker and Smith, 2010; Wiltgen et al., 2010). The need of using TIRF to guarantee a good axial resolution restricts the application of this approach to cells, as those of the human neuroblastoma SH-SY5Y cell line, where $\mathrm{IP}_{3} \mathrm{Rs}$ are close enough to the plasma membrane. In particular, it does not work in oocytes. A vast body 
of literature on $\mathrm{Ca}^{2+}$ signalsin this cell type exists which is still awaiting a clarification of the underlying $\mathrm{Ca}^{2+}$ and $\mathrm{IP}_{3} \mathrm{R}$ dynamics. Oocytes, on the other hand, are intact cells and can be used to explore changes in the morphology of the signals with maturation or with depth (Callamaras and Parker, 1999; Machaca, 2007). Thus, their study is of great interest. Unfortunately, fluorescence microscopy experiments in this cell type only give, so far, average (mean field) information of the underlying process. Mathematical tools have been widely used to link experimental observations at the level of puffs with the single channel activity.

As with experiments, there are different modeling approaches that differ on the spatial or time scale they try to resolve. Some models rely on the hypothesis that channels are in such close contact that the concentration of $\mathrm{Ca}^{2+}$ can be considered homogeneous throughout the cluster (Shuai and Jung, 2002, 2003b; Thul and Falcke, 2004; DeRemigio and Smith, 2005; Diambra and Guisoni, 2005; Ullah and Jung, 2006). This assumption allows for relatively fast simulations of cytosolic $\mathrm{Ca}^{2+}$ dynamics since $\mathrm{Ca}^{2+}$ diffusion within the cluster can be neglected. Other models inferred basic puff properties using a deterministic dynamics of spatially localized channels within a cluster combined with an analysis of fluorescence experiments (Shuai et al., 2006; Baran, 2007; Baran and Popescu, 2009; Bruno et al., 2010). These approaches have been useful as a first step for more elaborate analyses that incorporate the stochastic transitions between open and closed states of the channels. Some useful puff properties inferred with deterministic models include estimates of the current of underlying puffs, duration, and spatial width of the release sites.

We have recently analyzed experimental calcium puffs observed in Xenopus laevis oocytes using linescan experiments (Bruno et al., 2010). Using a previously developed backward algorithm (Ventura et al., 2005), we estimated the calcium flux that underlies fluorescent images. Since the algorithm is model independent, the estimates of Bruno et al. (2010) are independent of the dye or the exogenous buffer that are used in the experiments. The results we presented in Bruno et al. (2010) and those of Rose et al. (2006) could be explained by a model in which the total calcium current released through a cluster of $\mathrm{IP}_{3}$ Rs during a puff is a non-linear function of the number of open channels: linear when the number is small (between 1 and $\sim 20$ channels) and proportional to the square root of the number of channels for larger puffs. This type of scaling could be attributed to local $\mathrm{Ca}^{2+}$ depletion inside the ER. In fact, in Thul and Falcke (2004), the authors presented simulations of the current and concentration profiles generated by $\mathrm{Ca}^{2+}$ release from the ER and compared them with signal mass measurements in X. laevis oocytes. Taking into account both the luminal and cytosolic $\mathrm{Ca}^{2+}$ dynamics, they found that the release current was approximately proportional to the square root of the number of open channels. In view of these results, the change of scaling could be explained as follows (Bruno et al., 2010). Puffs of small amplitude correspond to very few open channels and relatively large distances from one another in which case depletion does not affect the single channel current. Puffs of large amplitude correspond to much smaller inter-channel distances in which case local depletion affects the luminal concentration in the vicinity of any other open channel and as a result, the total $\mathrm{Ca}^{2+}$ current scales as the square root of the number of channels.
Thul and Falcke (2004). However, these models do not describe the stochastic nature of the transitions between open and closed states of individual channels, so they can be viewed as mean field descriptions (Thul and Falcke, 2004; Bruno et al., 2010). Recently, the need of using stochastic and spatially resolved models to unveil the full repertoire of $\mathrm{Ca}^{2+}$ dynamics has been emphasized (Solovey et al., 2008; Thul et al., 2009; Rüdiger et al., 2010; Solovey and Ponce Dawson, 2010a,b; Diambra and Marchant, 2011; Thurley and Falcke, 2011).

In this work we compare mean field and stochastic puff models, with the latter including the description of individual channel openings and closings. In particular we show that the stochastic model reproduces the experimental observations of Bruno et al. (2010) with a fixed single channel current of $\sim 0.1 \mathrm{pA}$ regardless of the number of open channels. This implies that the instantaneous total current is proportional to the number of open IP $\mathrm{IP}_{3}$ at any given time. It is the average $\mathrm{Ca}^{2+}$ current released during the whole puff duration that scales non-linearly with the number of open or available channels, as does the (instantaneous) total current in the mean field models of Bruno et al. (2010), Thul and Falcke (2004). The studies of the present paper also show that in order to reproduce the experimental observations under the assumption that channels open and close simultaneously a smaller single $\mathrm{IP}_{3} \mathrm{R}$ current is needed than the actual one. Therefore, using mean field models to derive the underlying channel current from an image can lead to an underestimation of its value. The present study shows the limitations of mean field models and the importance of including a detailed description of the intra-cluster dynamics in order to infer realistic single channel properties from collective signals such as puffs.

The organization of the paper is as follows. In Section 2 we describe the models and the procedure to simulate puffs. Section 3 is divided in four parts. First we compare the time course of the current and fluorescence obtained with simulations of the mean field and stochastic puffs (see Section 3.1). In Section 3.2 we show a non-linear property of the system at the level of puffs, both in experimental data and simulations. In Section 3.3 we present the main result of this work which is the artifactual non-linearities that appear in mean field puff models and in mean field based analyses of experimental puffs. A simple semi-analytical model (see Section 3.4) helps to understand the results. Discussions and conclusions are presented in Section 4.

\section{MATERIALS AND METHODS}

\subsection{PUFF MODELS BASED ON A CLUSTER OF SPATIALLY LOCALIZED IP ${ }_{3}$ Rs}

We consider two kinds of models that differ mainly on the way we deal with the channel temporal dynamics. In mean field models, all available channels open and close simultaneously at times chosen a priori while in stochastic models the transitions between the open and closed conformations of the channels is stochastic and follows a channel kinetic model. The number and spatial distribution of the channels is based on the following evidence (Bruno et al., 2010): (a) puffs result from the release of $\mathrm{Ca}^{2+}$ through a few tens of $\mathrm{IP}_{3} \mathrm{Rs}$ within a cluster; (b) the size of the $\mathrm{Ca}^{2+}$ release region is $\sim 500 \mathrm{~nm}^{2}$, independent of the number of channels. As we describe in Section 2.1.2, we do not include the dynamics of $\mathrm{IP}_{3}$ binding and unbinding. Thus, by available channels we mean $\mathrm{IP}_{3}$ Rs with $\mathrm{IP}_{3}$ bound. 
Clusters consist of $N_{p}$ available channels $\left(N_{p} \leq 60\right)$ randomly distributed within a circle of radius $R=230 \mathrm{~nm}$ (stochastic models) or a square of side $\sim 250 \mathrm{~nm}$ (mean field models) at the beginning of the simulation. We only use cluster configurations that gave nearest neighbor channel distances larger than $40 \mathrm{~nm}$ in the stochastic simulations or $45 \mathrm{~nm}$ in the deterministic case.

The stochastic transitions between open and closed states of the channels occur at time scales much faster than the time scale that the experiments can resolve. For this reason, inferred values of the puff calcium current should be taken as averaged values. In contrast, in the simulations we know exactly how many channels are open with a resolution of $\sim 0.1 \mathrm{~ms}$, much better than the resolution with which we could infer the $\mathrm{Ca}^{2+}$ currents from the experimental records in Bruno et al. (2010). Therefore, in order to compare the maximum released current inferred from the experiments with the current used in the simulations we have to average the time course of the simulated current. This was done using a square moving average window.

\subsubsection{Mean field models}

The number of open channels, $N_{p}$, is constant during a puff and each open channel releases a constant $\mathrm{Ca}^{2+}$ current $I_{c h}$. However, the value of $I_{c h}$ may depend on $N_{p}$. We analyze two situations: the mean field linear model where $I_{c h}$ is independent of the number of open channels, in which case the total $\mathrm{Ca}^{2+}$ current is

$$
I_{L}\left(N_{p}\right)=I_{c h} N_{p}
$$

with $I_{c h}=0.1 \mathrm{pA}$ and the mean field non-linear model (Bruno et al., 2010) where $I_{c h}$ depends non-linearly on the number of open channels and the total $\mathrm{Ca}^{2+}$ current is given by:

$$
I_{N L}\left(N_{p}\right)=\left\{\begin{array}{lll}
I_{01} N_{p} & \text { if } & N_{p} \leq N_{p t} \\
I_{02} N_{p}^{1 / 2} & \text { if } & N_{p}>N_{p t}
\end{array}\right.
$$

with $I_{01}=0.017 \mathrm{pA}, I_{02}=0.08 \mathrm{pA}$, and $N_{p t}=22$. This expression corresponds to the one derived from the analysis of experimental puffs presented in Bruno et al. (2010).

\subsubsection{Stochastic models}

The single channel current is $0.1 \mathrm{pA}$, regardless of the number of open channels. We use this value for the current because it agrees with previous estimates of the type $1 \mathrm{IP}_{3} \mathrm{R}$ current in the ER membrane of X. laevis oocytes (Rose et al., 2006). It is also within the range of currents found recently for single recombinant type 3 IP 3 Rs (Vais et al., 2010).

The transition between the open and closed conformations of the channels is stochastic. We use two simple IP ${ }_{3} \mathrm{R}$ kinetic models which differ solely on the behavior of the mean open time as a function of $\left[\mathrm{Ca}^{2+}\right]$. We call these models $\mathbf{C I}$ (for which the mean open time is independent of $\left[\mathrm{Ca}^{2+}\right]$ ) and $\mathrm{CD}$ (for which the mean open time decreases with $\left.\left[\mathrm{Ca}^{2+}\right]\right)$. We use these models to test whether a $\mathrm{Ca}^{2+}$ dependent or a $\mathrm{Ca}^{2+}$ independent open to closed transition leaves a detectable signature on puffs or not. Both models assume that the channel can be in three states, closed (C), open $(\mathrm{O})$, or inhibited (I). The stochastic transitions between states occur according to the following schemes:

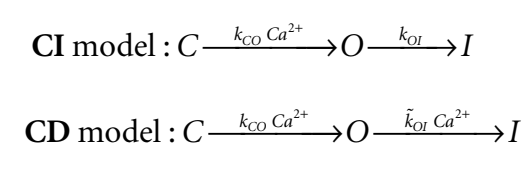

These models do not consider the kinetics of $\mathrm{IP}_{3}$ binding or unbinding to the $\mathrm{IP}_{3} \mathrm{R}$. According to most $\mathrm{IP}_{3} \mathrm{R}$ kinetic models, these processes occur so fast that the fraction of $\mathrm{IP}_{3}$ Rs with $\mathrm{IP}_{3}$ bound can be assumed to be fixed and given by an equilibrium relationship (Young and Keizer, 1992). In that sense, the kinetic scheme actually describes the transitions between states of the $\mathrm{IP}_{3} \mathrm{R}$ with $\mathrm{IP}_{3}$ bound. In addition, the kinetic schemes do not consider either that the channels can reopen once they enter the inhibited states. This simplification is reasonable to investigate basic puff properties. Namely, the time an $\mathrm{IP}_{3} \mathrm{R}$ remains inhibited under the conditions for which puffs are observed has been estimated as $\sim 2 \mathrm{~s}$ (Fraiman et al., 2006), a time much larger than the duration of a puff. This long inhibition time is compatible with the $\mathrm{IP}_{3} \mathrm{R}$ open probability obtained in reconstituted bilayer experiments (Bezprozvanny et al., 1991) but seems contradictory with the results of patch clamp experiments performed in isolated Xenopus oocyte nuclei using $\mathrm{K}^{+}$as the channel carrier (Mak et al., 1998). The latter type of experiments seems less disruptive than the former one since $\mathrm{IP}_{3} \mathrm{Rs}$ remain in a native membrane. However, the $\mathrm{Ca}^{2+}$ concentrations that are used on the luminal side of the channel in the patch clamp experiments are much lower than the ones encountered under physiological conditions. As discussed in Fraiman and Ponce Dawson (2004), this could explain the apparent contradiction between the long inhibition time estimated in Fraiman et al. (2006) and the observations of Mak et al. (1998). In fact, the existence of a broad variety of experimental results derived from single channel recordings calls for the definition of an optimal system where the source of the observed discrepancies can be unambiguously established (Foskett et al., 2007).

The values of the parameters of the kinetic models we consider in this paper are listed in Table 1. The open transition rate, $k_{C O}$, is taken from the De Young-Keizer $\mathrm{IP}_{3} \mathrm{R}$ model (Young and Keizer, 1992) and the transition rate from the open to the inhibited state is defined so that the mean open time of a single IP ${ }_{3} \mathrm{R}$ is $10 \mathrm{~ms}$ (a typical IP ${ }_{3} \mathrm{R}$ open duration; Mak et al., 1998). In the case of the CD model, we choose $\tilde{k}_{O I}$ so that $1 /\left(\tilde{k}_{O I} \mathrm{Ca}^{2+}\right)=10 \mathrm{~ms}$ with $\left[\mathrm{Ca}^{2+}\right]_{\text {mouth }}$ the value of $\left[\mathrm{Ca}^{2+}\right]$ at the mouth of an isolated open channel. We use $\left[\mathrm{Ca}^{2+}\right]_{\text {mouth }}=41.77 \mu \mathrm{M}$, an estimate obtained by numerical simulations assuming a single channel current $I_{c h}=0.1$ pA (Solovey et al., 2008). The transitions between states of the channels are computed using a discrete time Markov Chain procedure with a time step $1 \mu \mathrm{s}$.

We calculate the duration of the $\mathrm{Ca}^{2+}$ release during a puff as:

$$
t_{r}=\int_{0}^{t_{M}} 1_{\{n(t)>0\}} d t
$$

\section{Table 1 | Parameters of the $\mathrm{IP}_{3} \mathrm{R}$ models described in Section 2.1.2.}

\begin{tabular}{lll}
\hline Parameter & Value & Units \\
\hline$k_{\mathrm{CO}}$ & 20 & $\mu \mathrm{M}^{-1} \mathrm{~s}^{-1}$ \\
$k_{\mathrm{Ol}}$ & 100 & $\mathrm{~s}^{-1}$ \\
$\tilde{k}_{\mathrm{Ol}}$ & $100\left[\mathrm{Ca}^{2+}\right]_{\text {mouth }}^{-1}$ & $\mu \mathrm{M}^{-1} \mathrm{~s}^{-1}$ \\
{$\left[\mathrm{Ca}^{2+}\right]_{\text {mouth }}$} & 41.77 & $\mu \mathrm{M}$
\end{tabular}


where $1_{\{n(t)>0\}}=1$ if $n(t)>0$ and 0 otherwise, with $n(t)$ the number of open channels at time $t$ and $t_{M}=0.15 \mathrm{~s}$, the total time of the simulations. The $\mathrm{Ca}^{2+}$ release duration corresponds to the total time with at least one open channel.

We calculate the average $\mathrm{Ca}^{2+}$ current released as:

$$
I_{a v e}=\frac{\int_{0}^{t_{M}} I(t) d t}{\int_{0}^{t_{M}} 1 I_{n(t)>0} d t}=I_{c h} \frac{\int_{0}^{t_{M}} n(t) d t}{t_{r}}
$$

It corresponds to the current of a puff that releases the same total amount of $\mathrm{Ca}^{2+}$ but at a constant rate during the whole duration of the $\mathrm{Ca}^{2+}$ release. It is equivalent to a case where all channels of the cluster open and close simultaneously and release the same total amount of $\mathrm{Ca}^{2+}$ during the same time as the original puff.

\subsubsection{Simulations}

We solve numerically the set of coupled reaction-diffusion equations that result from the diffusion of cytosolic $\mathrm{Ca}^{2+}$ in the presence of localized $\mathrm{Ca}^{2+}$ channels. The details of the methods to simulate puffs are described in Solovey et al. (2008). Therefore, here we only present a brief description. Besides $\mathrm{Ca}^{2+}$, we consider the following species: an immobile endogenous buffer, a cytosolic fluorescent $\mathrm{Ca}^{2+}$ indicator $(B)$ and an exogenous mobile buffer. We use the same parameters we used in Solovey et al. (2008).

In the case of the mean field models, we use a fine spatial grid where individual channels are located. We use clusters of $1,3,5,10$, and 15 channels for the linear mean field model and 1, 3, 5, 10, 15, $20,25,30,40$, and 50 channels for the simulations with the nonlinear mean field model. The release duration was set to $18 \mathrm{~ms}$, the mean duration of $\mathrm{Ca}^{2+}$ release inferred from experiments in Bruno et al. (2010). In order to do a qualitative description of the differences between stochastic and mean field models (Section 3.1), we used long release durations ( $\sim 50 \mathrm{~ms}$ ), as illustrated in Figure 1.

To generate puffs with the stochastic model we use a simplified method also introduced in Solovey et al. (2008). The method is based on a scale separation, a similar approach as the one taken more recently by Skupin et al. (2010). Namely, we use a fine grid to determine the $\left[\mathrm{Ca}^{2+}\right]$ distribution within the cluster but describe its dynamics with a quasi-stationary approximation. In order to determine the contribution of each open channel to the $\left[\mathrm{Ca}^{2+}\right]$ distribution within the cluster, we first analyze this distribution when there is only one channel in the same cytosolic environment. The total $\left[\mathrm{Ca}^{2+}\right]$ within the cluster when there are several open channels is then approximated by a linear combination of the contributions due to each individual open channel. This detailed description of $\left[\mathrm{Ca}^{2+}\right]$ within the cluster is used to determine the openings and closings of the various channels. In this way we obtain the total $\mathrm{Ca}^{2+}$ current that is released from the cluster as a function of time. The spatiotemporal $\left[\mathrm{Ca}^{2+}\right]$ distribution outside the cluster is determined using a coarser grid in which the cluster is represented by a point source whose current is proportional to the number of open channels determined before. A reaction-diffusion system is solved on this coarser grid. We simulated 10 puffs for a cluster of a given number of channels that we vary between 10 and 50 .

The fluorescence $F$ is related to the concentration of $\mathrm{Ca}^{2+}$ ions bound to the fluorescent dye $(C a B)$ :

$$
F=\frac{\overline{[C a B]}}{[B]_{T}}\left(F_{\text {max }}-F_{\text {min }}\right)+F_{\text {min }}
$$

where $\overline{[C a B]}$ is an averaged version of $C a B$ due to the microscope point spread function (Bruno et al., 2010).

\subsection{SEMI-ANALYTICAL PUFF MODEL}

In order to gain a more intuitive understanding of the behaviors encountered with the numerical simulations, we also analyze a cartoon-like model that is amenable to analytic calculations. We consider the $\mathrm{IP}_{3} \mathrm{R}$ kinetic models introduced in Section 2.1.2 but the $\mathrm{Ca}^{2+}$ dynamics is simplified in two ways. First, at time zero all $N_{p}$ channels are open. All they can do is make a transition to the inhibited state. The puff ends when all channels are inhibited. This simplifies enormously the complexity of the $\mathrm{Ca}^{2+}$ dynamics, specially in the $\mathbf{C I}$ model, in which the transition from the open to the inhibited state is independent of $\mathrm{Ca}^{2+}$. To further simplify the dynamics of the $\mathrm{CD}$ model, we make a second approximation: all channels of the cluster are subject to the same $\left[\mathrm{Ca}^{2+}\right]$ which is proportional to the number of open channels $n$, so that the $\left[\mathrm{Ca}^{2+}\right]$ at time $t$ is $\left[\mathrm{Ca}^{2+}\right](t)=n(t)\left[\mathrm{Ca}^{2+}\right]_{\text {mouth }}$, where $\left[\mathrm{Ca}^{2+}\right]_{\text {mouth }}=41.77 \mu \mathrm{M}$ (Solovey et al., 2008) and $n=N_{p}, N_{p}-1, \ldots, 1,0$.

The two versions of the semi analytic model can be considered as extreme cases regarding the channels "coupling strength" (DeRemigio and Smith, 2005; Nguyen et al., 2005; Groff and Smith, 2008). The one with the $\mathbf{C I}$ kinetics corresponds to a fully uncoupled case where channels close stochastically and independently after a exponentially distributed open time with mean value $1 / k_{\text {Or }}$ The one with the CD kinetics, channels are fully coupled by cytosolic $\mathrm{Ca}^{2+}$.

The mean puff duration can be computed analytically for both models using Equation 4 and taking $t_{M}$ such that $n\left(t_{M}\right)=0$ for the first time (see details of the derivation in the Appendix). The result is that the mean duration of puffs that start with $N_{p}$ open channels is:

$$
\begin{gathered}
\left\langle t_{r}\right\rangle_{I}=\frac{1}{k_{O I}} \sum_{n=1}^{N_{p}} \frac{1}{n}, \\
\left\langle t_{r}\right\rangle_{D}=\frac{1}{k_{O I}} \sum_{n=1}^{N_{p}} \frac{1}{n^{2}},
\end{gathered}
$$

for models $\mathbf{C I}$ and $\mathbf{C D}$, respectively.

The average released current, $I_{\text {ave }}$, is given by Equation 5 . We do not have analytic expressions for the mean average current. Therefore, in order to compute it, we perform stochastic simulations of the semi analytic models (see Appendix for further details) that show that the mean average current can be approximated by the ratio of mean values:

$$
\left\langle I_{a v e}\left(N_{p}\right)\right\rangle \cong I_{c h} \frac{\left\langle\sum_{n=1}^{N_{p}} n \tau_{n}\right\rangle}{\left\langle t_{r}\right\rangle} \cong \frac{\sum_{n=1}^{N_{p}} n\left\langle\tau_{n}\right\rangle}{\sum_{n=1}^{N_{p}}\left\langle\tau_{n}\right\rangle}
$$

so that:

$$
\left\langle I_{\text {ave }}(N p)\right\rangle_{I} \cong \frac{I_{c h} N_{p}}{\sum_{n=1}^{N_{p}} \frac{1}{n}}
$$



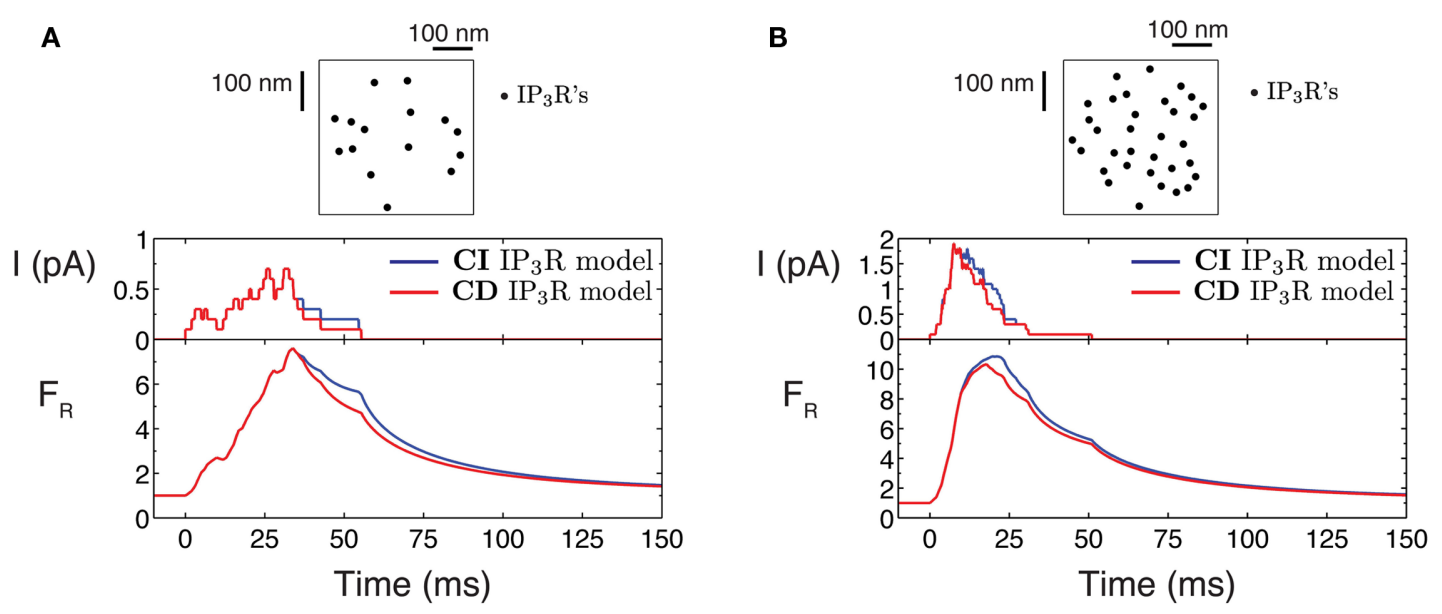

C
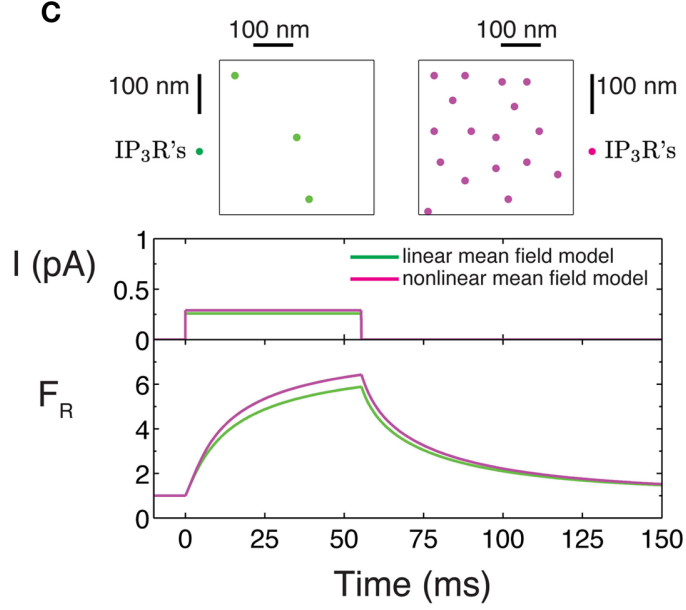

FIGURE 1 | Puff current and fluorescence time course for the stochastic and mean field models. (A,B) Stochastic puff model: simulations of the stochastic puff model for a cluster of $15 \mathrm{IP}_{3} R s(\mathbf{A})$ and $33 \mathrm{IP}_{3} R s(\mathbf{B})$. The spatial distribution of channels is shown in the upper square plots $(500 \mathrm{~nm} \times 500 \mathrm{~nm})$. The lower plots show the time evolution of the total current released and the resulting fluorescence signal. Red curves correspond to simulations in which the channels follow the $\mathbf{C D}$ model and blue lines, $\mathbf{C l}$ model. $\mathbf{( C , D )}$ Mean field puff

$$
\left\langle I_{a v e}(N p)\right\rangle_{D} \cong \frac{I_{c h} \sum_{n=1}^{N_{p}} \frac{1}{n}}{\sum_{n=1}^{N_{p}} \frac{1}{n^{2}}},
$$

D
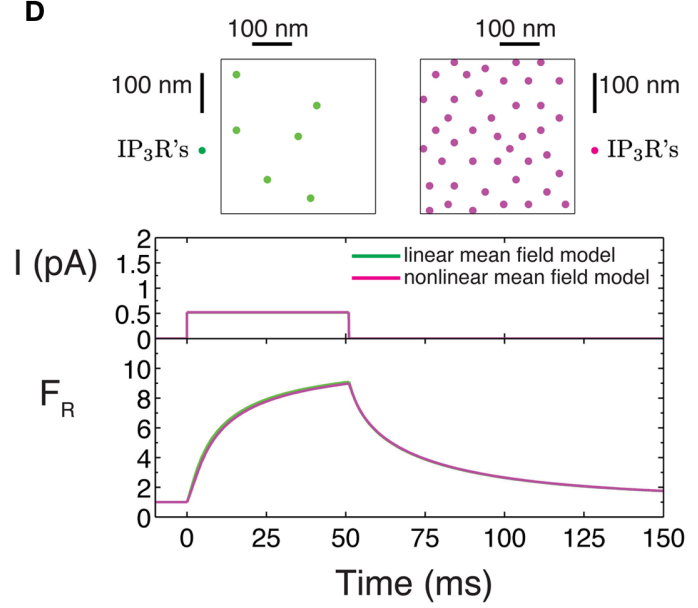

models: simulations of the mean field puff models. In (C) the simulations of the linear (green) and the non-linear (red) mean field models are such that the $\mathrm{Ca}^{2+}$ current is $\sim 0.25 \mathrm{pA}$. The number of channels are 3 in the linear mean field and 17 in the non-linear mean field model. In (D) the release current is $\sim 0.5 \mathrm{pA}$ and the number of channels is 6 (linear model) and 33 (non-linear model). The resulting fluorescence time course is shown in the bottom plot and the distribution of channels over a $500-n m \times 500-n m$ region is shown in the upper plot.

for models $\mathbf{C I}$ and $\mathbf{C D}$, respectively.

\section{RESULTS}

\subsection{TIME COURSE OF SIMULATED PUFFS}

Simulations can display the time course of puffs at different scales: from the single channel activity to the fluorescence signal. Useful parameters that characterize puffs are the amplitude $(A$, peak fluorescence) and the rising time ( $t_{p}$ time until the amplitude is reached). Qualitative differences between the stochastic and the mean field models are evident in Figure 1. In the stochastic case (Figures 1A,B), the current varies stepwise and the peak fluorescence is achieved before the end of $\mathrm{Ca}^{2+}$ release. However, in puffs

simulated with the mean field model the current is steady and the fluorescence increases during the total duration of $\mathrm{Ca}^{2+}$ release (Figures 1C,D).

The examples of Figures 1A,B are representative examples of two basic features observed in stochastic puffs: (a) the time to peak current shortens if the number of available channels increases and (b) the difference between puffs simulated with the $\mathbf{C I}$ and $\mathbf{C D} \mathrm{IP}_{3} \mathrm{R}$ model is not significant. There is a rapid recruitment of $\mathrm{IP}_{3} \mathrm{Rs}$ when $\mathrm{Ca}^{2+}$ release starts. This initial phase is followed by a usually larger falling phase in which channels close one by one, in agreement with Smith et al. (2009). All subsequent stochastic simulations will be done using model $\mathbf{C I}$ unless otherwise mentioned because the fluorescence signals of puffs simulated with the $\mathbf{C I}$ and $\mathbf{C D}$ models are statistically equivalent.

The amplitude of the puffs simulated with the non-linear mean field model is similar to the amplitudes of the puffs obtained with the stochastic model. The number of channels is also similar. However, 
the linear mean field model reproduces the same amplitude as the stochastic puffs but with significant fewer channels. The examples displayed in Figure 1 illustrate this difference.

Interestingly, the ratio of puff amplitudes is similar in all models when the number of channels is duplicated, as it is shown in Figure 1, comparing puffs from panels Figures 1A-D. This suggest that the ratio of amplitudes can give ambiguous results when trying to determine the "correct" puff underlying model.

As illustrated in Figures 1A,B, the rising time is shorter as more channels participate of the puff. This is a consequence of the fact that the coordination of channels due to CICR evoked by local $\left[\mathrm{Ca}^{2+}\right]$ elevations is more effective as the distance between $\mathrm{IP}_{3} \mathrm{Rs}$ decreases. Approximately $80 \%$ of the puffs simulated using the stochastic model are such that all available channels of the cluster participate although only approximately half of them were simultaneously open (data not shown). In the remaining $\sim 20 \%$ of the puffs, only $\sim 2$ channels did not open during the $150-\mathrm{ms}$ of the simulation time. This is more likely to occur in small clusters $\left(\left\langle\mathrm{N}_{\mathrm{p}} \sim 13\right\rangle\right)$ where the spacing between $\mathrm{IP}_{3} \mathrm{Rs}$ is relatively large and the coordination between channels due to CICR is less effective.

It is important to note that the average current and fluorescence time course of several stochastic simulations does not converge to the mean field counterpart. The mean field model assumes that all channels open and close simultaneously, therefore the release current is constant and the fluorescence increases during the puff.

\subsection{THE AMPLITUDE AND CURRENT OF PUFFS ARE NON-LINEARLY RELATED}

Observed puff amplitudes do not grow linearly with the maximum underlying current estimated from the observations (Bruno et al., 2010). Simulated puffs using the stochastic models show the same behavior, as shown in Figure 2. For large enough currents, the amplitude increases as the current increases, but at a lower rate than for small currents. One possible explanation to these observations is that the fluorescent dye is saturated at the position of the cluster due to the large amount of $\mathrm{Ca}^{2+}$ ions that are released during a puff. Nevertheless, this is not the case. Calibration experiments showed

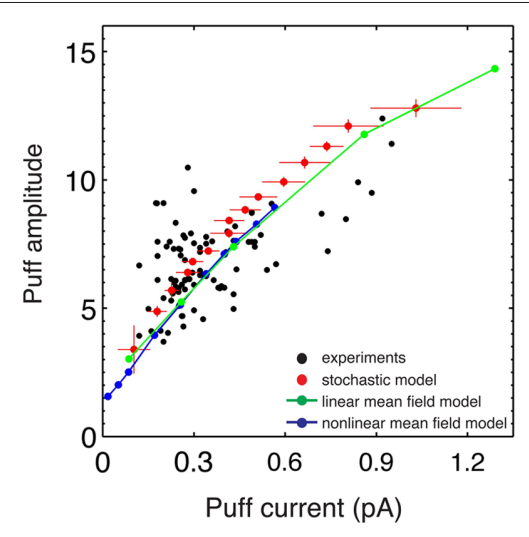

FIGURE 2 | Non-linear relationship between the amplitude and the release current. Puff amplitude as function of the maximum released current for experimental puffs (adapted from Bruno et al., 2010) and puffs simulated with the stochastic, linear and non-linear models. In the simulations of the stochastic puff model, $\mathrm{IP}_{3}$ Rs follow the $\mathbf{C D}$ kinetic mode. that dye saturation occurs at much larger amplitudes $\left(\mathrm{A} \sim F_{\max }\right.$ l $\left.F_{0}=48\right)$ than those of the puffs analyzed in Bruno et al. (2010). In the stochastic simulations, the $\mathrm{Ca}^{2+}$-bound dye concentration is never larger than $35 \mu \mathrm{M}$ (for $N_{p}=50$ ) while the total dye concentration is $40 \mu \mathrm{M}$. The reason for the observed non-linearity is the combination of the various processes that modulate the $\mathrm{Ca}^{2+}$-bound dye concentration within the volume corresponding to the pixel of the image. This processes depend on the kinetic rates of the reaction, the $\mathrm{Ca}^{2+}$ current, and the rate at which the $\mathrm{Ca}^{2+}$ bound dye leaves the volume due to diffusion.

Although puff amplitudes depend non-linearly on the underlying maximum currents, both for the simulations and for the experiments, the mean values of the puff current can be approximated by a function of the mean puff amplitudes with a relatively small dispersion. This indicates that, even if the interaction with the dye acts as a non-linear filter, the puff amplitude could eventually be used to estimate the underlying $\mathrm{Ca}^{2+}$ current.

\subsection{THE FINITE TIME RESOLUTION OF THE EXPERIMENTS FILTERS THE OBSERVATIONS DIFFERENTLY DEPENDING ON THE NUMBER OF OPEN CHANNELS}

We have analyzed in the previous Section the relationship between puff amplitude and the $\mathrm{Ca}^{2+}$ current that underlies the signal. We have shown, in particular, that these quantities depend non-linearly on one another in mean field models (linear and non-linear), stochastic models (independently of the $\mathrm{IP}_{3} \mathrm{R}$ kinetic scheme), and experiments. Even though the amplitude-current relationship obtained in all these cases is similar, we have observed that the number of channels involved in the linear and the non-linear mean field models are very different. In order to analyze this aspect in more detail, we now explore the dependence between number of channels and puff amplitude.

We show in Figure 3A a plot of puff amplitude as a function of the number of available channels, $N_{p}$, obtained with simulations of the stochastic puff model. We see that, as $N_{p}$ grows, the mean puff amplitude saturates. This indicates that the mean puff amplitude observed at a given cluster is not a good estimator of the number of channels of the cluster that can become open. We can distinguish an approximately linear regime in which the fluorescence signals contributed by each open channel summate linearly, in agreement with the recent results of Smith et al. (2009). Interestingly, these results show that, besides the non-linear filter that the $\mathrm{Ca}^{2+}$-dye reaction introduces between $\mathrm{Ca}^{2+}$ current and fluorescence, there is an additional source of non-linearity when going from the number of channels to puff amplitude. This additional source of non-linearity is related to the temporal coarse-graining imposed by the experimental temporal resolution acting on data that is characterized by different time courses depending on the size of the puffs. As shown in Figure 1, the time it takes for the current and the fluorescence to achieve their maximum values is shorter the larger is the puff (and the number of open channels that are involved). This means that the fluorescence that is measured experimentally is integrated over a shorter time interval. The dye response time and the finite time resolution of the experiments act as filters of this rapid increment producing the observed non-linear dependence. The fact that the time course is different depending on puff size is in turn related to the spatial organization of $\mathrm{IP}_{3} \mathrm{Rs}$ within the cluster. All models we 


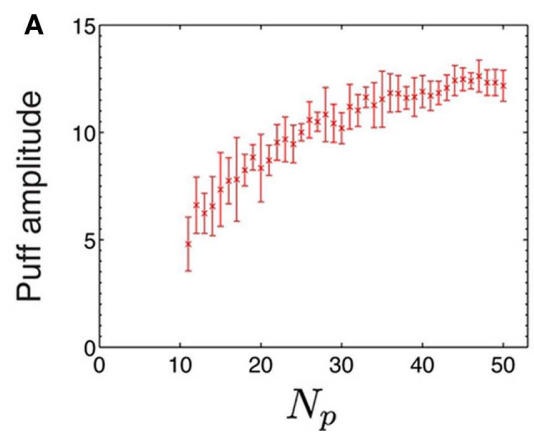

FIGURE 3 | Non-linearities as a function of the number of available channels. (A) Puff amplitude as a function of the number of available channels, $N_{p}$ for puffs simulated with the stochastic model and the $\mathbf{C D} I \mathrm{P}_{3} \mathrm{R}$ kinetic model. (B) Average $\mathrm{Ca}^{2+}$ current (Equation 5) released during a puff simulated using the stochastic model as a function of the number of available channels. The black

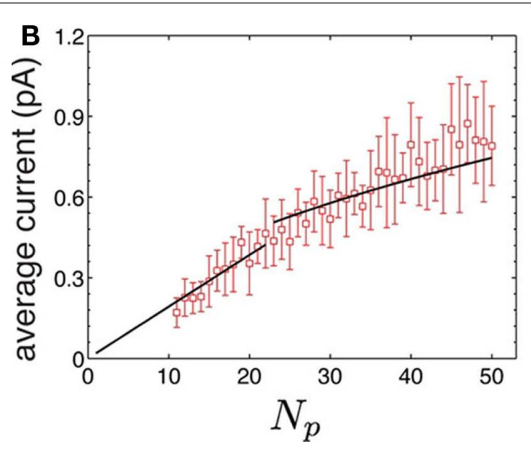

line corresponds to the best fit to a non-linear function of the form $I=I_{01} N_{p}$ for $N_{p} \leq 22$ and $I=I_{02} \sqrt{N_{p}}$ for $N_{p}>22$ which gives $I_{01}=0.019 \mathrm{pA}$ and $I_{02}=0.105 \mathrm{pA}$. $I_{01}$ should not identified as the actual single $I P_{3} R$ current but as an effective value that follows from the assumption that all channels open and close simultaneously. use in the simulations assume that the spatial cluster size is constant regardless of the number of channels that they contain. Thus, the simulated puffs that involve more open channels and have larger amplitudes, occur in clusters where the mean distance between available channels is shorter. This implies that CICR occurs on a faster time scale and the peak current is achieved within a shorter time. This non-linearity is absent in the linear mean field model.

In order to compare the new non-linearity that arises in the simulations of the stochastic puff model with the one that is intrinsic to the non-linear mean field model we compute the average current $\left(I_{a v e}\right.$, defined in Equation 5$)$ released during a puff simulated using the stochastic model. This average current corresponds to a puff that releases a constant current during the whole release duration (as if all $N_{p}$ channels of the cluster opened and closed simultaneously and released the same total amount of $\mathrm{Ca}^{2+}$ during the same time as the original puff). Therefore, it is equivalent to replacing the stochastic puff by one that could have been obtained with a mean field model. We found that the derivative of $I_{\text {ave }}\left(N_{p}\right)$ decreases as $N_{p}$ grows (Figure 3B). The deviation from linearity for large $N_{p}$ occurs although the simulations were performed with the stochastic model using a constant single channel current of $0.1 \mathrm{pA}$, regardless of the number of channels. The exact scaling between the average current and $N_{p}$ is not so relevant. However, it is remarkable that we can fit the data with the non-linear function $I_{\text {ave }}=I_{01} N p$ for $N_{p} \leq 22$ and $I_{\text {ave }}=I_{02} N_{p}^{1 / 2}$ for $N_{p} \leq 22$ (black line in Figure 3B). The square root scaling for large $N_{p}$ agrees with the value predicted in the simulations of Thul and Falcke (2004). From the fitting we find an effective single channel current for small $N_{p}$, $I_{01}=0.019 \mathrm{pA}$, which is five times smaller than the actual single channel current used in the simulations. Interestingly, it agrees with the value that the non-linear mean field model needed to reproduce the experimental observations (see Equation 2).

\subsection{UNDERSTANDING THE RESULTS USING THE SEMI-ANALYTICAL PUFF MODEL}

The fact that the effective single channel current is smaller than the actual one can be understood by considering two extreme (hypothetical) puffs with $N_{p}$ available channels and total release duration, $t_{r}$. In the first extreme case all $N_{p}$ channels open and close simultaneously. Thus, they all remain open during a time $t_{r}$. The corresponding average current is $I_{a v e}=I_{c h} N_{p} t_{r} / t_{r} I_{c h} N_{p}$, where $I_{c h}$ is the single channel current. In this case, $I_{\text {ave }}$ is a linear function of the number of channels that participate of the puff. The other extreme case is a puff in which the channels open and close sequentially so that there is one open channel at any given time. In this case, the average current is $I_{a v e}=I_{c h}$, independent of $N_{p}$. The simulations using the stochastic puff model give results in between these two extreme behaviors so that $I_{\text {eff }}=I_{\text {ave }} / N_{p} \leq I_{c h}$.

In order to get an intuitive understanding of why the slope between $I_{\text {ave }}$ and $N_{p}$ decreases with $N_{p}$ we simulated 500 puffs using the semi-analytical model (Section 2.2 ). We illustrate the nonlinear dependence between $I_{a v e}$ and $N_{p}$ for this model in Figure 4. In this case, we use both kinetic models of $\mathrm{IP}_{3} \mathrm{Rs}$ because they lead to different results. Although it is clearer for CD channels, in both cases the current grows non-linearly as $N_{p}$ increases. For example, in the CI case, $\left\langle I_{\text {ave }}\right\rangle \cong 0.4 \mathrm{pA}$ for $N_{p}=10$ and $\left\langle I_{\text {ave }}\right\rangle \cong 1 \mathrm{pA}$ for $N_{p}=40$. This non-linear relationship is equivalent to that of the non-linear mean field model. Results from simulations of the stochastic puff model that use the CD kinetic model lie in between the semi-analytical results for the two IP $\mathrm{I}_{3}$ models for $N_{p}>10$ (red squares and error bars in Figure 4A). The effective single channel current, $I_{e f f}=I_{a v e} / N_{p}$ of the semi-analytical models is plotted in Figure $4 \mathrm{~B}$ as a function of $N_{p}$. It is clear that as the number of channel increases, $I_{\text {eff }}$ approaches the value $\sim 0.02 \mathrm{pA}$, similarly to the puffs of the stochastic model.

The semi-analytical model allows us to go one step further in the analysis. We can use the approximation 8 to estimate $I_{\text {ave }}$. According to this expression, if the mean time with $n$ simultaneously open channels, $\left\langle\tau_{n}\right\rangle$, is independent of $n$, then the average current is linearly related to the number of open channels, $\left\langle I_{a v e}\right\rangle \cong I_{c h}\left(N_{p}+1\right) / 2$. However, in most cases $\left\langle\tau_{n}\right\rangle$ does depend on $n$. In particular, for the two $\mathrm{IP}_{3} \mathrm{R}$ kinetic models that we considered, $\mathrm{CI}$ and $\mathrm{CD}$, we obtain $\left\langle\tau_{n}\right\rangle^{3} \sim 1 / n$ and $\left\langle\tau_{n}\right\rangle \sim 1 / n^{2}$ respectively. Approximating $\sum_{n=1}^{N_{p}} \frac{1}{n} \sim \ln \left(N_{p}\right)$, which is good for large $N_{p}$, and taking into account that $\sum_{n=1}^{N_{p}} \frac{1}{n^{2}}=\frac{\pi^{2}}{6}$, we conclude that $\left\langle I_{\text {ave }}\right\rangle_{I} \sim \frac{N_{p}}{\ln N_{p}}$ and $\left\langle I_{\text {ave }}\left(N_{p}\right)\right\rangle_{D} \sim \ln$ $N_{p}$ for models $\mathbf{C I}$ and $\mathbf{C D}$, respectively. Therefore, we conclude that $I_{\text {ave }}^{p}$ is a non-linear function of $N_{p}$ with a slope that decreases with $N_{p}$. Within this view, the ability to approximate $I_{\text {ave }}$ by a linear function 


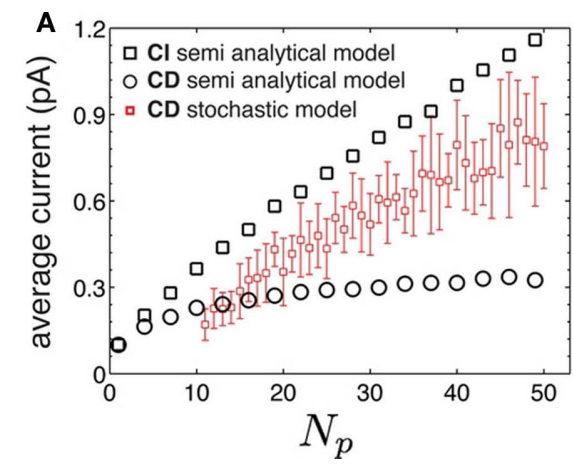

FIGURE 4 |The semi-analytical puff model supports the results of the stochastic puff model. (A) Average current as a function of $N_{\rho}$ for the semianalytical model and for simulated puffs obtained using the stochastic puff model with the $\mathbf{C D} I \mathrm{P}_{3} R$ kinetic model. (B) Effective single channel current as a function

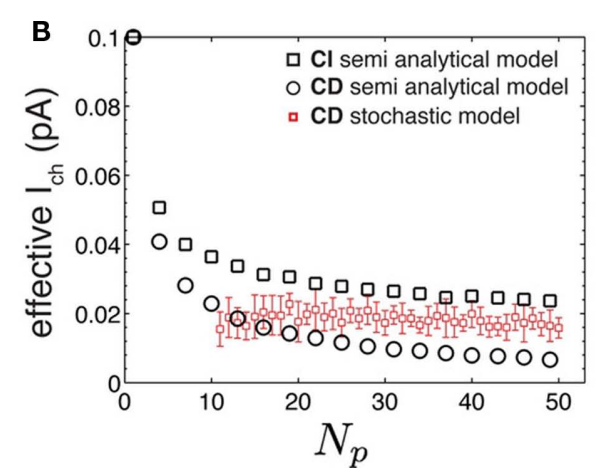

of $N_{p}$ for puffs simulated with the stochastic model using the $\mathbf{C D} I \mathrm{P}_{3} \mathrm{R}$ kinetic model. In (A,B), black circles and squares correspond to the semi-analytical model using the $\mathbf{C D}$ and the $\mathbf{C l}$ kinetic model, respectively. Small red squares and error bars represent mean and SD for puffs simulated with the stochastic model. for small $N_{p}$ and a relationship of the form $I_{a v e} \sim N_{p}^{1 / 2}$ instead of another non-linear function is only due to the limited interval of $N_{p}$ values over which we can observe the function. These estimates also show why $I_{\text {ave }}$ is smaller for the $\mathbf{C D}$ than for the $\mathbf{C I}$ model. In fact, the reason for this is that the transition from the open to the inhibited state is faster for the CD model and that the difference of time-scales increases with the number of channels that are simultaneously open. Thus, the state of the cluster with a large number of simultaneously open channels is shorter for the CD than for the CI model which results in a smaller average current.

\section{DISCUSSION}

Intracellular signals that involve $\mathrm{Ca}^{2+}$ release from the ER through clustered $\mathrm{IP}_{3} \mathrm{R}-\mathrm{Ca}^{2+}$ channels are crucial in a variety of cell types. A complete understanding of the processes involved in such signals requires a combination of experiments and mathematical modeling. Given the multiple time and spatial scales involved, mean field approaches are frequently used in models and data analysis. In a sense, data from fluorescence microscopy experiments is intrinsically of mean field type since it is averaged over the finite space and time resolution of the experimental method. In this work we have compared mean field approaches to stochastic models of $\mathrm{Ca}^{2+}$ puffs, a type of localized signal that involves $\mathrm{Ca}^{2+}$ release from several $\mathrm{IP}_{3} \mathrm{Rs}$ in a cluster and which has been observed in intact cells using optical techniques. We found that mean field models only match more accurate stochastic models under two unrealistic assumption: (a) a non-linear relationship between the current and the number of open channels and (b) a single channel current five times lower.

We have used two mean field models that we presented in a previous work (Bruno et al., 2010) where we showed that they approximate the inferred distribution of puff currents and the relationship between the puff amplitude and the underlying current. Both models assume that all available $\mathrm{IP}_{3} \mathrm{Rs}$ of the cluster open and close simultaneously. They differ on how the current depended on the number of channels: linear (Equation 1) and non-linear (Equation 2). We compared simulations of mean field models with simulations of a stochastic puff model that includes the stochastic transitions between open and close states of the channels (Solovey et al., 2008). In order to compare both models, we defined a mean field analog of the stochastic puffs as one with a constant current given by the average current released during the whole puff duration.

The first observation is that the amplitude of puffs is nonlinearly related to the released current, as it was shown in Bruno et al. (2010). This occurred although the fluorescent dye was not saturated. This result was also obtained with the simulations of the stochastic model (Figure 2). Only the non-linear mean field model was able to reproduce this non-linear relationship for the full range of the number of available channels considered here. The linear mean field model described the same behavior but using a much smaller number of channels than the number inferred from the experiments (Bruno et al., 2010; Figure 2).

Recent experimental results concluded that the total calcium current released through a cluster of $\mathrm{IP}_{3} \mathrm{Rs}$ during a puff is a nonlinear function of the number of open channels (Bruno et al., 2010): linear when the number is small and proportional to the square root of the number of channels for larger puffs. Although this type of scaling could be attributed to local $\mathrm{Ca}^{2+}$ depletion inside the ER (Thul and Falcke, 2004), we argue that this non-linear relationship occurs due to the assumed simultaneously action of the release sites intrinsic to mean field approaches. In fact, even though our stochastic simulations were done with a constant single channel current of $0.1 \mathrm{pA}$, we found that the average released current of stochastic puffs depended non-linearly on the number of available channels with a slope that decreased with the number of available channels (3B). Furthermore, we showed that the average current could be approximated by Equation 2 with $I_{01}$ approximately equal to the single channel current of the non-linear mean field model $(\sim 0.02 \mathrm{pA})$. This results implies that the mean field assumption also underestimates the single channel current by a factor of 5 (Figure 3 ).

We investigated the differences between stochastic and mean field approaches analytically as well. For this purpose, we used a simplified stochastic model to describe the dynamics of clustered $\mathrm{IP}_{3}$ Rs (semi-analytical model). Results using this model confirmed that the assumption that channels open and close simultaneously leads to an underestimation of the single $\mathrm{IP}_{3} \mathrm{R}$ channel current and that the relationship between the average current and the number of available channels is non-linear (Figure 4). 
In a previous work (Bruno et al., 2010), we have shown that the non-linear mean field model reproduces the puff to trigger amplitude distribution reported in Rose et al. (2006) better than the linear model. Our results shed light on why this is could be the case. Namely, the puff to trigger amplitude distribution does not take dynamics into account. However, the non-linear mean field model captures the additional non-linear filtering that the experiments impose. Indeed, it is remarkable that the relationship between puff amplitude and current obtained with the non-linear mean field model and the stochastic model conceded using the same range of number of available channels (Figure 2).

There is some controversy on how $\mathrm{IP}_{3}$ Rs behave (Bezprozvanny et al., 1991; Mak et al., 1998). Consequently, there is a variety of IP R kinetic models (Young and Keizer, 1992; Fraiman and Ponce Dawson, 2004; Shuai et al., 2007) and it is not completely clear which one describes the behavior of the channel under the conditions encountered in intact cells. In particular, it is not clear if the mean open time depends on $\mathrm{Ca}^{2+}$ or not. Therefore, in the case of the stochastic models, we did simulations using two simple kinetic models of the $\mathrm{IP}_{3} \mathrm{R}$ that only differed on whether the transition from the open to the closed state of the channel was $\mathrm{Ca}^{2+}$ dependent or not (CI and $\mathbf{C D}$ model respectively). The differences between both models are significant in the context of the semi-analytical model (Figure 4). This is easy to understand because the semi-analytical model maximized the differences between them. When using the CD model, the semi-analytical model is such that the $\left[\mathrm{Ca}^{2+}\right]$ sensed by each channel is additive and proportional to the number of open channels, as if all IP Rs were in the same spatial location. In contrast, when using the $\mathbf{C I}$ model, the underlying assumption is that the $\mathrm{Ca}^{2+}$ released by one channel has no influence on the other open channels. The real situation is somewhere in between both, as it is shown in Figure 4. In general, we did not found any significant difference between the puffs simulated with the stochastic model using any of the two kinetic $\mathrm{IP}_{3} \mathrm{R}$ models. We therefore conclude that this single channel property $\left(\mathrm{a} \mathrm{Ca}^{2+}\right.$ dependent or a $\mathrm{Ca}^{2+}$ independent open to closed transition) does not leave a detectable signature on puffs.

$\mathrm{Ca}^{2+}$ puffs are the result of a complicated underlying spatiotemporal dynamics. The relationship between puff amplitude and the underlying $\mathrm{Ca}^{2+}$ current can only be understood in terms of the interplay between the intensity and the dynamics of the release. The dynamics of the release, on the other hand, is strongly dependent on the spatial organization of the channels that become open. The combination of all these factors and the experimental limitations can lead to (unexpected) non-linear relationships. Modeling efforts that do not include the variability of the individual channel openings and closings provide averaged descriptions that are still valid. In particular, they can be used as building blocks of mathematical models of more global signals such as waves. However, if one is willing to understand the intra-cluster dynamics or to extract information on single channel kinetics from puff observations, a stochastic description is unavoidable.

\section{ACKNOWLEDGMENTS}

We acknowledge useful conversations with Ian Parker and Luciana Bruno. Guillermo Solovey would also like to acknowledge Marcelo Magnasco and the staff of the Center for Studies in Physics and Biology (The Rockefeller University). Daniel Fraiman and Silvina Ponce Dawson are members of the Carrera del Investigador Científico (CONICET). This research was supported by UBA (UBACyT X145) and CONICET (PIP 11220080101612).

\section{REFERENCES}

Baran, I. (2007). Characterization of local calcium signals in tubular networks of endoplasmic reticulum. Cell Calcium 42, 245-260.

Baran, I., and Popescu, A. (2009). A model-based method for estimating $\mathrm{Ca} 2+$ release fluxes from linescan images in Xenopus oocytes. Chaos 19, 037106.

Berridge, M. J., Lipp, P., and Bootman, M. D. (2000). The versatility and universality of calcium signalling. Nat. Rev. Mol. Cell Biol. 1, 11-21.

Bezprozvanny, I., James, W., and Ehrlich, B. E. (1991). Bell-shaped calciumresponse curves of $\operatorname{lns}(1,4,5) \mathrm{P} 3$ - and calcium-gated channels from endoplasmic reticulum of cerebellum. Nature 351, 751-754.

Bootman, M., Niggli, E., Berridge, M., and Lipp, P. (1997). Imaging the hierarchical Ca2+ signalling system in HeLa cells. J. Physiol. (Lond.) 499, 307-314.

Bruno, L., Solovey, G., Ventura, A. C., Dargan, S., and Dawson, S. P. (2010). Quantifying calcium fluxes underlying calcium puffs in Xenopus laevis oocytes. Cell Calcium 47, 273-286.
Callamaras, N., Marchant, J. S., Sun, X.-P., and Parker, I. (1998). Activation and co-ordination of InsP3-mediated elementary $\mathrm{Ca} 2+$ events during global $\mathrm{Ca} 2+$ signals in Xenopus oocytes. J. Physiol. (Lond.) 509, 81-91.

Callamaras, N., and Parker, I. (1994). Inositol 1,4,5-trisphosphate receptors in Xenopus laevis oocytes, localization and modulation by $\mathrm{Ca} 2+$. Cell Calcium 15, 66-78.

Callamaras, N., and Parker, I. (1999). Radial localization of inositol 1,4,5-trisphosphate-sensitive ca $2+$ release sites in xenopus oocytes resolved by axial confocal linescan imaging. J. Gen. Physiol. 113, 199-213.

Cohen, J. E. (2004). Mathematics is biology's next microscope, only better; biology is mathematics' next physics, only better. PLoS Biol. 2, e439. doi: 10.1371/journal.pbio.0020439

Cox, D. R. (1962). Renewal Theory. London: Methuen.

DeRemigio, H., and Smith, G. D. (2005). The dynamics of stochastic attrition viewed as an absorption time on a terminating Markov chain. Cell Calcium $38,73-86$.
Diambra, L., and Guisoni, N. (2005). Modeling stochastic $\mathrm{Ca} 2+$ release from a cluster of IP3-sensitive receptors. Cell Calcium 37, 321332.

Diambra, L., and Marchant, J. S. (2011) Inositol (1,4,5)-trisphosphate receptor microarchitecture shapes $\mathrm{ca}(2+)$ puff kinetics. Biophys. J. 100, 822-831.

Finch, E. A., Turner, T. J., and Goldin, S M. (1991). Calcium as a coagonist of inositol 1,4,5-trisphosphate-induced calcium release. Science 252, 443-446.

Foskett, J. K., White, C., Cheung, K. H., and Mak, D.-O. D. (2007). Inositol triphosphate receptor $\mathrm{Ca} 2+$ release channels. Physiol. Rev. 87, 593-658.

Fraiman, D., Pando, B., Dargan, S., Parker, I., and Dawson, S. P. (2006). Analysis of puff dynamics in oocytes, interdependence of puff amplitude and interpuff interval. Biophys. J. 90, 3897-3907.

Fraiman, D., and Ponce Dawson, S. (2004). A model of the IP_3 receptor with a luminal calcium binding site, stochastic simulations and analysis. Cell Calcium 35, 403-413.

Groff, J. R., and Smith, G. D. (2008). Calcium-dependent inactivation and the dynamics of calcium puffs and sparks. J. Theor. Biol. 253, 483-499.

Iino, M. (1990). Biphasic Ca2+ dependence of inositol 1,4,5-trisphosphateinduced Ca release in smooth muscle cells of the guinea pig taenia caeci. $J$. Gen. Physiol. 95, 1103-1122.

Machaca, K. (2007). Ca2+ signaling differentiation during oocyte maturation. J. Cell. Physiol. 213, 331-340.

Mak, D.-O. D., and Foskett, J. K. (1997). Single-channel kinetics, inactivation, and spatial distribution of inositol trisphosphate (IP3) receptors in Xenopus oocyte nucleus. J. Gen. Physiol. 109, 571-587.

Mak, D.-O. D., McBride, S., and Foskett, J. K. (1998). Inositol 1,4,5-trisphosphate activation of inositol tris-phosphate receptor $\mathrm{Ca} 2+$ channel by ligand tuning of $\mathrm{Ca} 2+$ inhibition. Proc. Natl. Acad. Sci. U.S.A. 95, 15821-15825.

Nguyen, V., Mathias, R., and Smith, G. D. (2005).A stochastic automata network descriptor for Markov chain models of instantaneously coupled intracellular Ca2+ channels. Bull. Math. Biol. 67, 393-432. 
Parker, I., Choi, J., and Yao, Y. (1996). Elementary events of InsP3-induced Ca2 liberation in Xenopus oocytes, hot spots, puffs and blips. Cell Calcium 20, 105-121.

Parker, I., and Smith, I. F. (2010). Recording single-channel activity of inositol trisphosphate receptors in intact cells with a microscope, not a patch clamp. J. Gen. Physiol. 136, 119-127.

Patel, S., Joseph, S. K., and Thomas, A. P. (1999). Molecular properties of inositol 1,4,5-trisphosphate receptors. Cell Calcium 25, 247-264.

Ramos-Franco, J., Fill, M., and Mignery, G. A. (1998). Isoform-specific function of single inositol 1,4,5-trisphosphate receptor channels. Biophys. J. 75, 834-839.

Rose, H. J., Dargan, S., Shuai, J., and Parker, I. (2006). “Trigger” events precede calcium puffs in Xenopus oocytes. Biophys. J. 91, 4024-4032.

Rüdiger, S., Nagaiah, C., Warnecke, G., and Shuai, J. W. (2010). Calcium domains around single and clustered IP3 receptors and their modulation by buffers. Biophys. J. 99, 3-12.

Shuai, J., Pearson, J. E., Foskett, J. K., Mak, D. O., and Parker, I. (2007). A kinetic model of single and clustered IP_3 receptors in the absence of $\mathrm{Ca} 2+$ feedback. Biophys. J. 93, 1151-1162.

Shuai, J., Rose, H. J., and Parker, I. (2006). The number and spatial distribution of IP3 receptors underlying calcium puffs in Xenopus oocytes. Biophys. J. 91, 4033-4044.

Shuai, J.W., and Jung, P. (2003a). Optimal ion channel clustering for intracellular calcium signaling. Proc. Natl. Acad. Sci. U.S.A. 100, 506-510.

Shuai, J. W., and Jung, P. (2003b). Subthreshold Ca2+ waves. New J. Phys. 5,132 .

Shuai, J.-W., and Jung, P. (2002). Stochastic properties of $\mathrm{Ca} 2+$ release of inositol 1,4,5-trisphosphate receptor clusters. Biophys. J. 83, 87-97.

Skupin, A., Kettenmann, H., and Falcke, M. (2010). Calcium signals driven by single channel noise. PLoS Comput. Biol. 6, e1000870. doi: 10.1371/journal.pcbi. 1000870

Smith, I. F., and Parker, I. (2009). Imaging the quantal substructure of single IP3R channel activity during Ca2+ puffs in intact mammalian cells. Proc. Natl. Acad. Sci. U.S.A. 106, 6404-6409.

Smith, I. F., Wiltgen, S. M., Shuai, J., and Parker, I. (2009). Ca(2+) puffs originate from preestablished stable clusters of inositol trisphosphate receptors. Sci. Signal. 2, ra77.

Solovey, G., Fraiman, D., Pando, B., and Ponce Dawson, S. (2008). Simplified model of cytosolic $\mathrm{Ca}[\sup 2+]$ dynamics in the presence of one or several clusters of $\mathrm{Ca}[\sup 2+]$-release channels. Phys. Rev. E Stat. Nonlin. Soft Matter Phys. 78, 041915.

Solovey, G., and Ponce Dawson, S. (2010a). Intra-cluster percolation of calcium signals. PLoS ONE 5, e8997. doi: 10.1371/journal.pone.0008997

Solovey, G., and Ponce Dawson, S. (2010b). Observable effects of Ca2+ buffers on local Ca2+ signals. Phil. Trans. R. Soc. Lond. A 368, 5597-5603.

Sun, X.-P., Callamaras, N., Marchant, J. S., and Parker, I. (1998). A continuum of InsP3-mediated elementary Ca2+ signalling events in Xenopus oocytes. J. Physiol. (Lond.) 509, 67-80.

Taylor, C. W. (1998). Inositol trisphosphate receptors, $\mathrm{Ca} 2+-$ modulated intracellular ca2+ channels. Biochim. Biophys. Acta 1436, 19-33.

Thul, R., and Falcke, M. (2004). Release currents of IP3 receptor channel clusters and concentration profiles. Biophys. J. 86, 2660-2673.

Thul, R., Thurley, K., and Falcke, M. (2009). Toward a predictive model of $\mathrm{Ca} 2+$ puffs. Chaos 19, 037108.

Thurley, K., and Falcke, M. (2011). Derivation of $\mathrm{Ca} 2+$ signals from puff properties reveals that pathway function is robust against cell variability but sensitive for control. Proc. Natl. Acad. Sci. U.S.A. 108, 427-432.

Ullah, G., and Jung, P. (2006). Modeling the statistics of elementary calcium release events. Biophys. J. 90, 3485-3495.

Vais, H., Foskett, J. K., and Daniel Mak, D.-O. (2010). Unitary $\mathrm{Ca}(2+)$ current through recombinant type $3 \mathrm{InsP}(3)$ receptor channels under physiological ionic conditions. J. Gen. Physiol. 136, 687-700.

Ventura, A. C., Bruno, L., Demuro, A. Parker, I., and Dawson, S. P. (2005). A model-independent algorithm to derive $\mathrm{Ca} 2+$ fluxes underlying local cytosolic Ca2+ transients. Biophys. J. 88, 2403-2421.

Wiltgen, S. M., Smith, I. F., and Parker, I. (2010). Superresolution localization of single functional ip3r channels utilizing ca2+ flux as a readout. Biophys. J. $99,437-446$.
Yao, Y., Choi, J., and Parker, I. (1995) Quantal puffs of intracellular Ca2+ evoked by inositol trisphosphate in Xenopus oocytes. J. Physiol. (Lond.) 482, 533-553.

Yao, Y., and Parker, I. (1991). Regenerative release of calcium from functionally discrete subcellular stores by inositol trisphosphate. Proc. Biol. Sci. 246, 269-274.

Young, G. W. D., and Keizer, J. (1992). A single-pool inositol 1,4,5-trisphosphate-receptor-based model for agonist-stimulated oscillations in $\mathrm{Ca} 2+$ concentration. Proc. Natl. Acad. Sci. U.S.A. 89, 9895-9899.

Conflict of Interest Statement: The authors declare that the research was conducted in the absence of any commercial or financial relationships that could be construed as a potential conflict of interest.

Received: 01 June 2011; accepted: 21 July 2011; published online: 01 August 2011. Citation: Solovey G, Fraiman D and Dawson SP (2011) Mean field strategies induce unrealistic non-linearities in calcium puffs. Front. Physio. 2:46. doi: 10.3389/fphys.2011.00046

This article was submitted to Frontiers in Computational Physiology and Medicine, a specialty of Frontiers in Physiology.

Copyright (c) 2011 Solovey, Fraiman and Dawson. This is an open-access article subject to a non-exclusive license between the authors and Frontiers Media SA, which permits use, distribution and reproduction in other forums, provided the original authors and source are credited and other Frontiers conditions are complied with. 


\section{APPENDIX}

\section{SEMI-ANALYTICAL PUFF MODEL}

We describe here the derivation of the formulas for the mean duration of the puff in the semi-analytical model, Equation $7 \mathrm{~b}$, the variance of the duration which we use to calculate effective current in Figure 4B, the simulation procedure to obtain the average current, Equation eq: stoch.metodos.semianalitico.Iave, and the approximate solutions, Equations eq: stoch.metodos.semianalitico. Iavemedio.

eq: stoch.metodos.semianalitico.Iavemedio

In this model we consider that the puff starts with $N_{p}$ open channels that may inhibit at any time with a rate $\mu$ that, in principle, may depend on the number of open channels $n$. After $t=0$, the number of channels decreases stepwise, one by one, so the puff duration is the sum of the time elapsed with $N_{p}$ open channels $\left(\tau_{N_{p}}\right)$, plus the time elapsed with $N_{p}-1$ open channels $\left(\tau_{N_{p}-1}\right)$ and so on until the last channel closes. Therefore, the duration of the $\mathrm{Ca}^{2+}$ release, Equation 4, can be expressed as:

$$
t_{r}=\sum_{n=1}^{N_{p}} \tau_{n}
$$

In Equation A.1, $n$ represents the number of simultaneously open channels and $\tau_{n}$ the time elapsed with $n$ open channels. The mean puff duration, $\left\langle t_{r}\right\rangle=\sum_{n=1}^{N_{p}}\left\langle\tau_{n}\right\rangle$, can be easily calculated because $\tau_{n}$ 's are exponential random variables. In fact, the time each channel remains open, given that there are $n$ open channels $[T(i \mid n), i=1, \ldots, n]$, is exponentially distributed with a mean value $\mu^{-1}$ (which may depend on $n$, depending on the IP ${ }_{3} \mathrm{R}$ model). The number of simultaneously open channels reduces to $n-1$ when the first one out of $n$ channels inhibits. Therefore the time elapsed with $n$ open channels is the minimum among $n$ exponential random variables:

$$
\tau_{n}=\min (T(1 \mid n), T(2 \mid n), \ldots, T(n \mid n))
$$

The minimum between $n$ exponential random variables is also exponentially distributed but with mean value $\left\langle\tau_{n}\right\rangle=n \mu^{-1}$. Finally, the mean $\mathrm{Ca}^{2+}$ release duration of a cluster of $N_{p}$ channels is:

$$
\left\langle t_{r}\right\rangle=\sum_{n=1}^{N_{p}} \frac{1}{n \mu(n)},
$$

Equation A.3 is valid for any $\mu(n)$. Replacing $\mu$ by the corresponding open to inhibited transition rate, it reduces to Equations 7b. We consider two models as in Section 2.1.2. If the channels of the cluster follow the CI model, they behave independently and close stochastically after a mean dwell time $\left(k_{O I}^{-1}\right)$, so $\mu=\mu_{i}=k_{O r^{*}}$ On the other hand, model CD considers that IP ${ }_{3}$ Rs are coupled by $\mathrm{Ca}^{2+}$, so that $\mathrm{Ca}^{2+}$ release from one channel affects the inhibiting probability of the rest opened channels. Different levels of coupling were considered in Groff and Smith (2008), DeRemigio and Smith (2005), Nguyen et al. (2005), where it is assumed that the $\left[\mathrm{Ca}^{2+}\right]$ sensed by an open channel is $\left[\mathrm{Ca}^{2+}\right]_{\text {mouth }}+(n-1) c^{*}$, where $n$ is the number of open channels, $c^{*}$ is the coupling strength and $\left[\mathrm{Ca}^{2+}\right]_{\text {mouth }}$ is the $\left[\mathrm{Ca}^{2+}\right]$ value at the mouth of one isolated open channel. In most of this paper, we work with two extreme cases, $c^{*}=0($ model $\mathbf{C I})$ and $c^{*}=\left[\mathrm{Ca}^{2+}\right]_{\text {mouth }}($ model $\mathrm{CD})$. Therefore, for model CD, $\mu=\mu_{d}=\tilde{k}_{\text {OI }} n\left[\mathrm{Ca}^{2+}\right]_{\text {mouth }}$ (see Section 2.1.2). In addition, according to Section 2.1.2 and Table 1, the open to inhibit transition rate is chosen such that the mean open time of a single channel is the same for both models, $\tilde{k}_{O I}\left[\mathrm{Ca}^{2+}\right]_{\text {mouth }}=k_{O I}$, and therefore $\mu_{d}(n)=k_{O I} n$. The assumption underlying the expression for $\mu_{d}$ is that the channels are at the same spatial location and $\left[\mathrm{Ca}^{2+}\right]$ is additive.

The variance of the puff duration can be obtained using Equation A.1 and the fact that $\tau_{n}$ are independent exponentially distributed random variables. Therefore:

$$
\begin{aligned}
\operatorname{Var}\left(t_{r}\right) & =\sum_{n=1}^{N_{p}} \operatorname{Var}\left(\tau_{n}\right) \\
& =\sum_{n=1}^{N_{p}}\left(\left\langle\tau_{n}^{2}\right\rangle-\left\langle\tau_{n}\right\rangle^{2}\right) \\
& =2 \sum_{n=1}^{N_{p}} \frac{1}{n^{2} \mu(n)^{2}}-\left(\sum_{n=1}^{N_{p}} \frac{1}{n \mu(n)}\right)^{2}
\end{aligned}
$$

It is also possible to calculate the distribution function of $t, F(t)$. Since $t_{r}$ is the sum of $N_{p}$ exponentially distributed random variables $\left(\tau_{n}, n=1, \ldots, N_{p}\right)$ with different mean values $\left[\left\langle\tau_{n}\right\rangle=(n \mu)^{-1}\right]$, following (Cox, 1962) the distribution function is:

$$
F(t)=1-\sum_{n=1}^{N_{p}} A_{n} \exp (-n \mu(n) t),
$$

where $A_{n}=\prod_{i=1 ; i \neq j}^{N_{p}} i \mu(i) /(i \mu(i)-n \mu(n))$.

The average current released during a puff, Equation 5, cannot be easily obtained analytically. Therefore, we performed simple simulations of the semi-analytical model to calculate $I_{\text {ave }}$. The simulation procedure is as follows. From Equation A.1 the average current can be expressed as:

$$
I_{a v e}=I_{c h} \frac{\sum_{n=1}^{N_{p}} n \tau_{n}}{t_{r}},
$$

where $t_{r}$ is the total release time given by Equation A.1. Therefore, the puff is a random process that is fully defined by $\tau_{n}\left(n=1, \ldots, N_{p}\right)$ and each $\tau_{n}$ is the minimum of $n$ exponential random variables, Equation A.2. So we numerically generate $n$ exponentially distributed random numbers with mean $\mu^{-1}$ (in the case of the CI model, $\mu=\mu_{i}=k_{O I}$ and in the case of the CD model, $\left.\mu=\mu_{d}=k_{O I} n\right)$ and take the minimum between them which we define as $\tau_{n}$. Repeating this operation for each $n$ completes the random process. With these values, we calculate the total release time given by Equation A.1 and the average current given by Equation A.6. Repeating this random process 500 times for each $N_{p}$ we obtain the mean $I_{a v e}$ as a function of $N_{p}$ that we plot in Figure 4A.

The approximate solutions in Equation 9b are obtained calculating the ratio of mean values in Equation A.6. Although this is not true in general, the results of the simulations show that the relative error of those approximations is always below 15\% and decreases with $N_{p}$ (for the CI model) and between 20 and 25\% (for the CD model) for the values of $N_{p}$ considered. 